See discussions, stats, and author profiles for this publication at: https://www.researchgate.net/publication/334692681

\title{
Asymmetric natural convection flow in three dimensional attic space
}

Conference Paper in AIP Conference Proceedings · July 2019

DOI: $10.1063 / 1.5114485$

CITATIONS

4 authors, including:
READS

18

Suvash C. Saha

University of Technology Sydney

185 PUBLICATIONS 1,204 CITATIONS

SEE PROFILE

Some of the authors of this publication are also working on these related projects:

Project Thermal Engineering View project

A 3D NUMERICAL MODEL FOR FINE PARTICULATES DEPOSITION AND CLEARANCE IN THE HUMAN RESPIRATORY TRACT View project 


\title{
Asymmetric natural convection flow in three dimensional attic space
}

\author{
Huimin $\mathrm{CUI}^{1}{ }^{1}$ ), Feng $\mathrm{XU}^{2,3}$ b), Suvash C. SAHA ${ }^{4 c)}$, and Qingkuan $\mathrm{Liu}^{5 \mathrm{~d})}$ \\ ${ }^{I}$ Department of Mathematics and Physics, Shijiazhuang Tiedao University, Shijiazhuang 050043, China \\ ${ }^{2}$ School of Civil Engineering, Beijing Jiaotong University, Beijing 100044, China \\ ${ }^{3}$ Beijing's Key Laboratory of Structural Wind Engineering and Urban Wind Environment, Beijing 100044, China \\ ${ }^{4}$ School of Mechanical and Mechatronic Engineering, Faculty of Engineering and Information Technology, \\ University of Technology Sydney, Ultimo NSW 2007, Australia \\ ${ }^{5}$ Wind Engineering Research Center, Structure Health Monitoring and Control Institute, Shijiazhuang Tiedao \\ University, Shijiazhuang 050043, China \\ ${ }^{a)}$ Corresponding author: huimincui @ stdu.edu.cn \\ b)fxu@bjtu.edu.cn \\ ${ }^{c}$ Suvash.Saha@uts.edu.au \\ d)lqk@stdu.edu.cn
}

\begin{abstract}
Natural convection in an attic space with top cooling is investigated by three dimensional numerical simulation for a wide range of Rayleigh numbers. The numerical results show the development of natural convection flow in attic space. Three stages: an initial stage, a transitional stage and a fully developed stage are involved. At higher Rayleigh numbers, the flow in the attic space is three dimensional and asymmetry with respect to geometric symmetry plane. The critical Rayleigh number for asymmetric flow is found.
\end{abstract}

\section{INTRODUCTION}

Natural convection is extensively present in an attic space, which has close relation with the thermal comfort of occupants in buildings and the energy cost for heating and air-conditioning ${ }^{[1]}$. Accordingly, increasing studies focus on natural convection in an attic space.

The boundary conditions including day-time top heating and night-time top cooling were usually applied for natural convection in an attic space in most studies ${ }^{[2,3]}$. For the day-time top heating condition, natural convection flows remain steady and laminar as the Rayleigh number smaller or equal to $4.9 \times 10^{7}$, and heat transfer in the enclosure is mainly through conduction. Under the day-time top heating condition, heat and mass transfer is much smaller than that under the night-time top cooling condition.

Under the night-time top cooling condition, transient natural convection usually involves an initial stage, a transitional stage and a fully developed stage. The flow state is determined by the aspect ratio, the Rayleigh number and the Prandtl number ${ }^{[4,5]}$. In the initial stage, the thermal boundary layer forms in the proximity of the bottom or the top and the thickness increases with time during which conduction dominates heat transfer. As time increases, convection increases and transient natural convection enters the transitional stage. The distinct intrusion can travel on the bottom wall and the starting plume can ascend in the center. Finally, natural convection approaches a steady or an unsteady state. The literature review indicates that the dynamics of transient natural convection under the night-time top cooling condition has been discussed and the corresponding fundamental scales have been obtained ${ }^{[6]}$. However, the three dimensional flow regime in quasi-steady state is not clear, especially for the asymmetric flow. 
In this paper, transient natural convection flow with Prandtl number 0.7 is described following sudden top cooling and bottom heating. In quasi-steady stage, the asymmetric flow structure determined by the aspect ratio and the Rayleigh number, is characterized. The critical Rayleigh number for the transition between symmetric flow and asymmetric flow regimes, which are illustrated in a Ra-I figure, has been obtained.

\section{Computational Models and Numerical Procedures}

The dimension of the enclosure is a height of $H=0.024 \mathrm{~m}$, a width of $W=0.048 \mathrm{~m}$ and the half length of $L=$ $0.048 \mathrm{~m}$, respectively. The aspect ratio defined by $H / L$ is 0.5 . A schematic of the enclosure is illustrated in Fig. 1. The tiny tips of the bottom corners are cut approximately $4 \% L$ to avoid a singularity at the joint point between top and bottom walls in numerical simulation. Such a slight change has insignificant influence on natural convection in the enclosure ${ }^{[6,7]}$.

The working fluid here is air $(\operatorname{Pr}=0.7)$ and initially motionless at a uniform temperature of $T_{0}=0$. The top wall of the enclosure is initially suddenly cooled to $T_{\mathrm{c}}=-0.5$, the bottom wall is heated to $T_{\mathrm{h}}=0.5$, and the front and back vertical walls as well as the two tiny tips are adiabatic. Additionally, the condition of rigid and no-slip is applied on all wall boundaries.

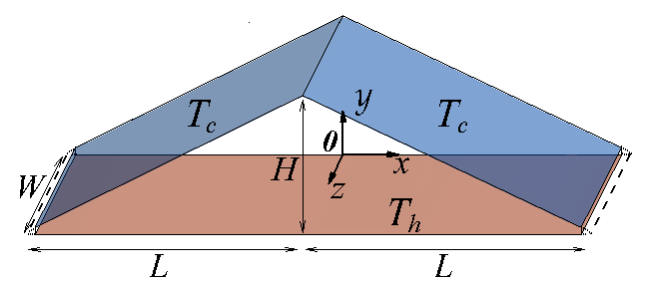

FIGURE 1. Schematic of an attic space for the aspect ratio of 0.5

Three dimensional Navier-Stokes equations and energy equation with Boussinesq approximation describe well transient natural convection in an enclosure ${ }^{[8]}$. The governing equations may also be non-dimensionalized using the following scales: $x, y, z \sim H ; t \sim H^{2} /\left(\kappa \mathrm{Ra}^{1 / 2}\right) ;\left(T-T_{0}\right) \sim\left(T_{\mathrm{h}}-T_{\mathrm{c}}\right) ; u, v, w \sim \kappa \mathrm{Ra}^{1 / 2} / H ;$ and $\rho^{-1} \partial p / \partial x, \rho^{-1} \partial p / \partial y, \rho^{-1} \partial p / \partial z$ $\sim \kappa^{2} \mathrm{Ra} / H^{3}$, which are expressed as:

$$
\begin{gathered}
\frac{\partial u}{\partial x}+\frac{\partial v}{\partial y}+\frac{\partial w}{\partial z}=0 \\
\frac{\partial u}{\partial t}+u \frac{\partial u}{\partial x}+v \frac{\partial u}{\partial y}+w \frac{\partial u}{\partial z}=-\frac{\partial p}{\partial x}+\frac{\operatorname{Pr}}{\operatorname{Ra}^{1 / 2}}\left(\frac{\partial^{2} u}{\partial x^{2}}+\frac{\partial^{2} u}{\partial y^{2}}+\frac{\partial^{2} u}{\partial z^{2}}\right) \\
\frac{\partial v}{\partial t}+u \frac{\partial v}{\partial x}+v \frac{\partial v}{\partial y}+w \frac{\partial v}{\partial z}=-\frac{\partial p}{\partial y}+\frac{\operatorname{Pr}}{\operatorname{Ra}^{1 / 2}}\left(\frac{\partial^{2} v}{\partial x^{2}}+\frac{\partial^{2} v}{\partial y^{2}}+\frac{\partial^{2} v}{\partial z^{2}}\right)+\operatorname{Pr} T \\
\frac{\partial w}{\partial t}+u \frac{\partial w}{\partial x}+v \frac{\partial w}{\partial y}+w \frac{\partial w}{\partial z}=-\frac{\partial p}{\partial z}+\frac{\operatorname{Pr}}{\operatorname{Ra}^{1 / 2}}\left(\frac{\partial^{2} w}{\partial x^{2}}+\frac{\partial^{2} w}{\partial y^{2}}+\frac{\partial^{2} w}{\partial z^{2}}\right) \\
\frac{\partial T}{\partial t}+u \frac{\partial T}{\partial x}+v \frac{\partial T}{\partial y}+w \frac{\partial T}{\partial z}=\frac{1}{\operatorname{Ra}^{1 / 2}}\left(\frac{\partial^{2} T}{\partial x^{2}}+\frac{\partial^{2} T}{\partial y^{2}}+\frac{\partial^{2} T}{\partial z^{2}}\right)
\end{gathered}
$$

Transient natural convection in the enclosure is dependent on three nondimensional parameters, which are the aspect ratio $(A)$, the Rayleigh number $(\mathrm{Ra})$ and the Prandtl number $(\mathrm{Pr})$, defined as:

$$
\operatorname{R} a=\frac{g \beta\left(T_{h}-T_{c}\right) H^{3}}{v \kappa}, \operatorname{Pr}=\frac{v}{\kappa}, A=\frac{H}{L} .
$$

We adopt the finite volume method to discretize of the equations (i)-(v) and the SIMPLE scheme to solve the pressure-velocity coupling problem. A second-order center-differenced scheme was employed to discretize second derivative and linear first derivative terms and a QUICK scheme was used for advection terms. Additionally, the 
time integration terms were discretized using a second-order difference scheme. Under-relaxation factors were used in the iteration of the discretized equations.

For the discretization of the computational domain, a non-uniform mesh was built. In this study, the dependence test of the mesh and the time step was performed for the largest Rayleigh number of $\mathrm{Ra}=10^{7}$. The three meshes $(L \times H \times W)$ of $110 \times 30 \times 41,141 \times 41 \times 51$ and $171 \times 51 \times 61$ and the two time steps of 0.01 and 0.02 were tested. The results are presented in Table 1 . Note that the variation between the Nusselt numbers of the bottom wall is relative to those calculated using the mesh of $141 \times 41 \times 51$ and the time step of 0.02 . The Nusselt number averaged in the fully developed stage is defined as

$$
\mathrm{Nu}=\frac{1}{S_{w}} \iint_{S_{w}} \frac{\partial T}{\partial n} d S
$$

Under consideration of computing cost and the variation between the results obtained using different meshes and time steps, the mesh of $141 \times 41 \times 51$ and the time step of 0.02 were adopted in this paper.

TABLE 1. Results of mesh and time step test for $A=0.5, \operatorname{Pr}=0.7$

\begin{tabular}{cccc}
\hline Number & $\begin{array}{c}\text { Mesh } \\
\boldsymbol{L} \times \boldsymbol{H} \times \boldsymbol{W}\end{array}$ & Time Step & $\mathbf{R a}=\mathbf{1 0}$, Variation of Nu $(\%)$ \\
\hline 1 & $110 \times 30 \times 41$ & 0.02 & 1.75 \\
2 & $141 \times 41 \times 51$ & 0.02 & - \\
3 & $171 \times 51 \times 61$ & 0.02 & 0.41 \\
4 & $141 \times 41 \times 51$ & 0.01 & 0.30 \\
\hline
\end{tabular}

\section{Natural Convection Flows}

A range of Rayleigh numbers from $10^{3}$ to $10^{6}$ was considered. The development of transient flow and flow state in the quasi-steady stage have been observed.

\section{Development of natural convection flows}

Table 2 shows the temperature iso-surfaces at different times for $\operatorname{Pr}=0.7, A=0.5$ and $\operatorname{Ra}=10^{6}$. The bule temperature iso-surface $T=-0.3$ denotes the cooled flow, and the red temperature iso-surface $T=0.3$ denotes the heated flow. In initial stage, the flow is laminar, as seen at $t=2$. In transition stage, the intrusion flows form near the bottom, as seen at $t=8$. The plumes have formed in the transition stage, which is clearly at $t=25$. In the quasisteady stage, three dimensional flow in the attic space was asymmetry, as seen at $t=2800$.

TABLE 2. Temperature iso-surfaces at different times for $\operatorname{Pr}=0.7, A=0.5$ and $\mathrm{Ra}=10^{6}$. The bule temperature iso-surface is $T=$ -0.3 , and the red temperature iso-surface is $T=0.3$

\begin{tabular}{llll}
\hline $\operatorname{Pr}$ & $t=2$ & $t=8$ & $t=25$ \\
\hline
\end{tabular}

Flow structures in the fully developed stage

Table 3 shows temperature iso-surfaces and streamlines in the triangular cavity at $t=2800$ in the fully developed stage for $A=0.5$. At lower Rayleigh numbers, the flow in steady (or quasi-steady) stage is laminar and symmetric, which is similar to the case of $\mathrm{Ra}=10^{3}$. As Rayleigh number increasing, the flow becomes asymmetric. It's 
approximate two-dimensional, as seen at $\mathrm{Ra}=10^{4}$. At higher Rayleigh numbers, the three dimensional flow is asymmetric, the transverse rolls and longitudinal rolls are clear.

TABLE 3. Temperature iso-surfaces and streamlines in attic space at $t=2800$ in the fully developed stage for $A=0.5$. The bule is the temperature iso-surface of $T=-0.3$ and the red is the temperature iso-surface of $T=0.3$

\begin{tabular}{cccccc}
\hline Type & $\mathrm{Ra}=10^{3}$ & $\mathrm{Ra}=10^{4}$ & $\mathrm{Ra}=10^{5}$ \\
\hline \\
Tsomperature
\end{tabular}

The degree of symmetry $I$ for different Rayleigh numbers shows that the transitional Rayleigh number for asymmetry flow is $9 \times 10^{3}$.

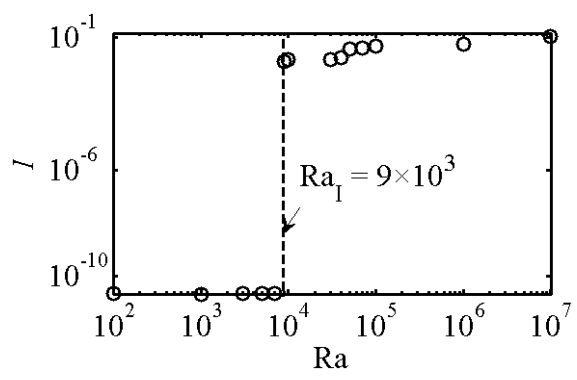

FIGURE 2. Degree of symmetry $I$ for different Rayleigh numbers

\section{CONCLUSIONS}

The development of natural convection flows in an attic space under night-time top cooling condition is numerically investigated. The 3D numerical simulations at higher Rayleigh numbers were performed. The asymmetry flow in an attic space was observed, and the critical Rayleigh number for asymmetry flow was found.

\section{REFERENCES}

1. A. Ben-Nakhi, M. A. Mahmoud, Appl. Therm. Eng., vol. 28, pp. 1522-1535(2008).

2. D. Poulikakos, A. Bejan, J. Fluid Mech., vol. 131, pp. 251-269(1983).

3. S. C. Saha, J. C. Patterson, C. Lei, Int. J. Heat Mass Transfer, vol. 46, pp. 621-638(2010).

4. S. C. Saha, Energy Build., vol. 43, pp. 2908-2917(2011).

5. H. Cui, F. Xu, S. C. Saha, Int. J. Therm. Sci., vol. 111, pp. 330-339(2017).

6. S. C. Saha, M. M. K. Khan, Energy Build., vol. 43, pp. 2564-2571(2011).

7. C. Lei, J. C. Patterson, Int. J. Heat Mass Transfer, vol. 46, pp. 1183-1197(2003).

8. H. W. Muller, M. Tveitereid, S. Trainoff, Phys. Rev. E, vol. 48, pp. 263-272(1993). 